American Journal of Neuroscience 2 (1): 17-27, 2011

ISSN 1948-9900

C 2011 Science Publications

\title{
Improved Application of Paraconsistent Artificial Neural Networks in Diagnosis of Alzheimer's Disease
}

\author{
${ }^{1}$ Helder F.S. Lopes, ${ }^{2}$ Jair M. Abe, ${ }^{1-3}$ Paulo A.M. Kanda,${ }^{4,6}$ Sergio Machado, \\ ${ }^{4}$ Bruna Velasques, ${ }^{4}$ Pedro Ribeiro, ${ }^{5}$ Luis F.H. Basile, \\ ${ }^{3}$ Ricardo Nitrini and ${ }^{1-3}$ Renato Anghinah \\ ${ }^{1}$ Department of Behavioral and Cognitive Disorders, \\ Faculty of Medicine, University of Sao Paulo, \\ Rua Arruda Alvim, 206, Sao Paulo, SP, CEP 05410020, Brazil \\ ${ }^{2}$ Department of Production Engineering, \\ ICET-Paulista University of Sao Paulo, \\ Brazil/Institute for Advanced Studies, Brazil \\ ${ }^{3}$ Department of Neurology, \\ Faculty of Medicine, University of Sao Paulo, Brazil \\ ${ }^{4}$ Institute of Psychiatry, \\ Brain Mapping and Sensory Motor Integration, \\ Federal University of Rio de Janeiro (IPUB/UFRJ), Brazil \\ ${ }^{5}$ Psychophysiology Laboratory, \\ University Metodista of Sao Paulo, Brazil / High-Resolution EEG Section, \\ Division of Neurosurgery, Faculty of Medicine-University of Sao Paulo, Brazil \\ ${ }^{6}$ Institute of Psychiatry, \\ Panic and Respiration Laboratory, \\ Federal University of Rio de Janeiro (IPUB/UFRJ), Brazil
}

\begin{abstract}
Problem statement: The visual analysis of Electroencephalogram (EEG) activity has shown useful as a complementary tool in Alzheimer Disease (AD diagnosis) when the diagnosis remains uncertain, in addition to be used in some clinical protocols. However, this analysis is subject to the inherent equipment imprecision, biological artifact, electrical records, and subjective physician interpretation of the visual analysis variation. The Artificial Neural Network (ANN) could be a helpful tool, appropriate to address problems such as prediction and pattern recognition. Approach: In this study, it was used a new class of ANN, namely the Paraconsistent Artificial Neural Network (PANN), which is capable of handling uncertain, inconsistent, and paracomplete information, for recognizing predetermined patterns of EEG activity and to assess its value as a possible complementary method for AD diagnosis. Thirty three AD patients and thirty four controls patients of EEG records were obtained during relaxed wakefulness. It was considered as normal patient pattern, the background EEG activity between 8.0 $\mathrm{Hz}$ and $12.0 \mathrm{~Hz}$ (with an average frequency of $10.0 \mathrm{~Hz}$ ), allowing a range of $0.5 \mathrm{~Hz}$. Results: The PANN was able to recognize waves that belonging to their respective bands of clinical use (theta, delta, alpha, and beta), leading to an agreement with the clinical diagnosis at $80 \%$ of sensitivity and at $73 \%$ of specificity. Conclusion: Supported by results, the PANN could be a promising tool to manipulate EEG analysis, bearing in mind the following considerations: the growing interest of specialists in EEG visual analysis and the ability of the PANN to deal in directly imprecise, inconsistent and paracomplete data, providing an interesting quantitative and qualitative analysis.
\end{abstract}

Key words: Electroencephalogram, alzheimer's disease, pattern recognition, artificial neural network, paraconsistent logic, Paraconsistent Artificial Neural Network (PANN), EEG visual analysis, paracomplete data, qualitative analysis

Corresponding Author: Helder F.S. Lopes, Reference Center of Behavioral and Cognitive Disorders of Faculty of Medicine, University of São Paulo. Rua Arruda Alvim, 206, São Paulo, SP, CEP 05410020, Brazil 


\section{INTRODUCTION}

Several studies on behavioral and cognitive neurology have been conducted to characterize dementias through biological and functional markers, for instance, the Electro Encephalo Graphic (EEG) activity, aimed at understanding the evolution of Alzheimer Disease (AD), following its progression, as well as leading toward better diagnostic criteria for early detection of cognitive impairment (Machado et al., 2010; Duffy et al., 2011). At present, there is no method able to determine a definitive diagnosis of dementia, where a combination of tests would be necessary to obtain a probable diagnosis.

The EEG activity is a record of brain's electrical activity, providing a space-time representation of synchronic postsynaptic potentials. The main generating sources of these electrical fields are most likely perpendicular in relation to the cortical surface, such as in the cortical pyramidal neurons. With regard to EEG visual analysis, several studies have shown that it is useful in aiding AD diagnosis, being indicated in some clinical protocols. During the relaxed awake state, normal EEG in adults is predominantly composed by the alpha band frequency, which is generated by interactions of the slum-cortical and thalamocortical systems. Incidentally, the most common finding in EEG visual analysis is the slowing of the brain electrical activity compounds regarding delta and theta rhythms, and the decreasing or absence of the alpha rhythm. However, these findings are more common in moderate and advanced stages of AD.

Most of the theories and techniques available for the analysis of quantitative EEG are based on classical logic (Puri and Li, 2010) and, therefore, have inherent limitations to this logic. Although several theories have been developed in order to overcome these limitations, e.g. fuzzy set theory, Rough theory, non-monotonic reasoning, among others, cannot deal with inconsistencies and paracompleteness, at least directely. Thus, it is needed a new kind of logic to deal with uncertain, inconsistent and paracomplete data (Silva Filho et al., 2010).

The Artificial Neural Network (ANN) can be described as a computational system consisting of a set of highly interconnected processing elements, called artificial neurons, which process information in response to external stimuli. An artificial neuron is a simplistic representation that emulates the signal integration and the behavior of the firing threshold of biological neurons by means of mathematical structures. Artificial neurons, like their biological counterparts, are bound together by connections that determine the information flow among neurons. Stimuli are transmitted from the processing element to another one via synapses or interconnections, which can be excitatory or inhibitory. Neural networks have an advantage over conventional programming because they lie in their ability to solve problems that do not have an algorithmic solution or where the available solution is too complex to be found (Syan and Harnarinesingh, 2010). Thus, neural networks are well suited to tackle problems that people are good at solving, such as prediction and pattern recognition. Moreover, ANNs have been applied within the medical domain for clinical diagnosis, imaging analysis and interpretation, signal analysis and interpretation (Karait et al., 2009; Syan and Harnarinesingh, 2010), and drug development. Therefore, ANN constitutes an interesting tool for EEG qualitative analysis. On the other hand, in EEG analysis we are faced with imprecise, inconsistent and paracomplete data. In order to manipulate this information directly, recently, some interesting theories have been proposed: fuzzy sets and rough sets for example.

In this study, we employed a particular kind of ANN based on Paraconsistent Annotated Evidential Logic E $\tau$ (Abe and Nakamatsu, 2009), which is capable of manipulating imprecise, inconsistent and paracomplete data in order to make a first study of the recognition of EEG standards with the aim of using it in $\mathrm{AD}$ diagnosis. In the methodology section, we will present this new artificial neural network, the Paraconsistent Artificial Neural Networks (PANN) (Silva Filho et al., 2010).

In this study we aim to continue our previous studies (Abe et al., 2011; Lopes et al., 2009), in order to improve the performance of PANN on the classification of patients with $\mathrm{AD}$ likely, using as criterion for classifying the slowing of brain activity based on the patients.

\section{MATERIALS AND METHODS}

The atomic formulas of the logic $\mathrm{E} \tau$ are of the type $\mathrm{p}(\mu, \lambda)$, where $(\mu, \lambda) \in[0,1] 2$ and $[0,1]$ is the real unitary interval (p denotes a propositional variable). $\mathrm{P}(\mu, \lambda)$ can be intuitively read: "It is assumed that p's favorable evidence is $\mu$ and contrary evidence is $\lambda$ Thus:

- $\mathrm{p}(1.0,0.0)$ can be read as a true proposition

- $\mathrm{p}(0.0,1.0)$ can be read as a false propositio

- $\mathrm{p}(1.0,1.0)$ can be read as an inconsistent proposition 
- $\mathrm{p}(0.0,0.0)$ can be read as a paracomplete (unknown) proposition

- $\mathrm{p}(0.5,0.5)$ can be read as an indefinite proposition

We introduce the following concepts (Abe and Nakamatsu, 2009) (all considerations are taken with $0 \leq$ $\mu, \lambda \leq 1$ :

- Uncertainty degree (Eq. 1)

- Certainty degree (Eq. 2)

- Complementation (Eq. 3)

$\mathrm{G}_{\mathrm{un}}(\mu, \lambda)=\mu+\lambda-1$

$\mathrm{G}_{\mathrm{ce}}(\mu, \lambda)=\mu-\lambda$

$\mathrm{X}_{\mathrm{c}(\mathrm{y})}=1-\mathrm{Y}$

An order relation is defined on $[0,1]^{2}:\left(\mu_{1}, \lambda_{1}\right) \leq$ $\left(\mu_{2}, \lambda_{2}\right) \Leftrightarrow \mu_{1} \leq \mu_{2}$ and $\lambda_{2} \leq \lambda_{1}$ constituting a lattice that will be symbolized by $\tau$.

With the uncertainty and certainty degrees we can achieve the following 12 output states (Fig. 1): Extreme states that are, false, true, inconsistent and paracomplete, and non-extreme states (Table 1).

Some additional control values are:

- $\quad \mathrm{Vc}_{\mathrm{ic}}=$ maximum value of uncertainty control $=\mathrm{Ft}_{\mathrm{ct}}$

- $\quad \mathrm{Vc}_{\mathrm{ve}}=$ maximum value of certainty control $=\mathrm{Ft}_{\mathrm{ce}}$

- $\quad \mathrm{Vc}_{\mathrm{pa}}=$ minimum value of uncertainty control = $\mathrm{Ft}_{\mathrm{ct}}$

- $\quad \mathrm{Vc}_{\mathrm{fa}}=$ minimum value of certainty control $=-\mathrm{Ft}_{\mathrm{ce}}$

For the discussion in the present paragraph we have used: $\mathrm{Ft}_{\mathrm{ct}}=\mathrm{Ft}_{\mathrm{ce}}=0.5$.

In the PANN the main aim is to ascertain how to determine the certainty degree concerning a proposition, i.e., if it is false or true. To this end, we take into account the certainty degree $\mathrm{G}_{\mathrm{ce}}$. The uncertainty degree $\mathrm{G}_{\mathrm{un}}$ indicates the 'measure' of the inconsistency or par completeness. If the certainty degree is low or the uncertainty degree is high, it generates an in definition.

Using the concepts of basic Para consistent Artificial Neural Cell (PANC-Fig. 2), we can obtain the family of PANC considered in this study, as described in Table 2.

We analyzed 67 EEGs records, 34 normal's and 33 probable $\mathrm{AD}$ (Table 3), during the awake state at rest (i.e., eyes closed). We used electrodes placed according to the 10-20 international system and an EEG 32 channels EMSA device, with $200 \mathrm{~Hz}$ sample frequency.

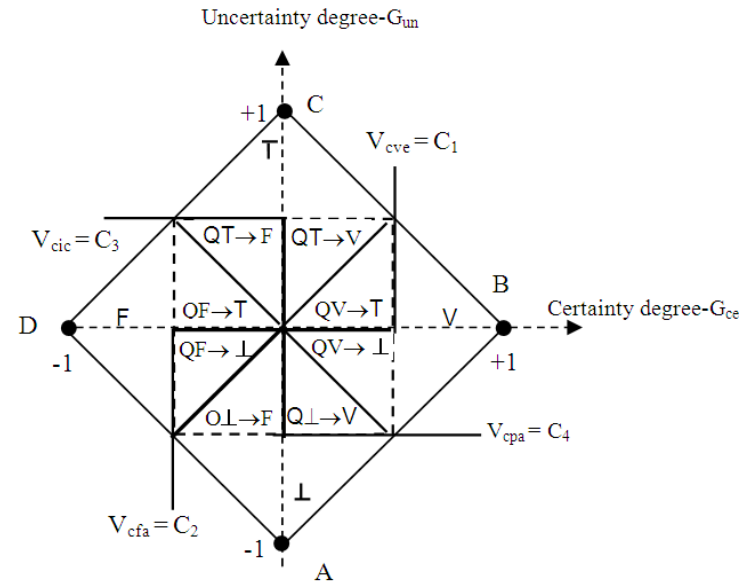

Fig. 1: The figure displays the output regions of the lattice, constituting the decision-making of the inputs. In this lattice we have 12 output states: extreme and non-extreme states. Table 1 for symbology. $\mathrm{C}_{1}=\mathrm{Vc}_{\mathrm{ve}}=$ truth control value; $\mathrm{C}_{2}=$ $\mathrm{Vc}_{\mathrm{fa}}=$ falsity control value; $\mathrm{C}_{3}=\mathrm{Vc}_{\mathrm{ic}}=$ inconsistency control value; $\mathrm{C}_{4}=\mathrm{Vc}_{\mathrm{pa}}=$ paracompleteness control value

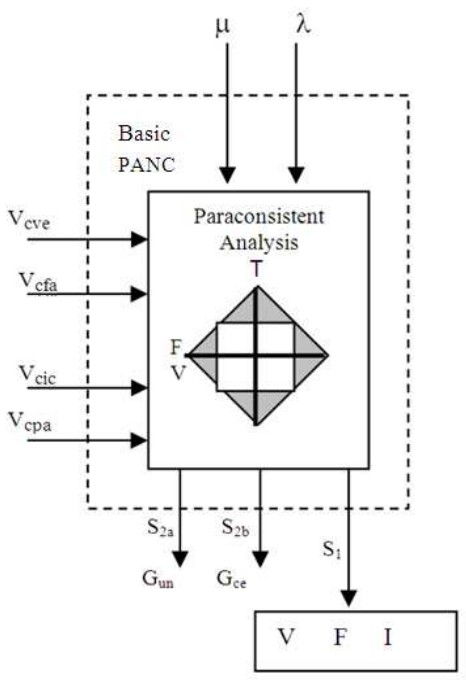

Fig. 2: Basic cell of PANN. $\mu=$ input of favorable evidence; $\lambda=$ input of contrary evidence; $\mathrm{T}=$ inconsistent; $\perp=$ paracomplete; $\mathrm{V}=$ true; $\mathrm{F}=$ false; $\mathrm{V}_{\text {cve }}=$ truth control value; $\mathrm{V}_{\text {cfa }}=$ falsity control value; $\mathrm{V}_{\text {cic }}=$ inconsistency control value; $\mathrm{V}_{\mathrm{cpa}}=$ paracompleteness control value; $\mathrm{S}_{2 \mathrm{a}}=$ output with uncertainty degree $\mathrm{G}_{\mathrm{un}} ; \mathrm{S}_{2 \mathrm{~b}}$ $=$ output with certainty degree $\mathrm{G}_{\mathrm{ce}} ; \mathrm{S}_{1}=$ output with true $(\mathrm{V})$, false $(\mathrm{F})$ or indefinite constant (I) 
Am. J. Neuroscience 2(1): 17-27, 2011

Table 1: Extreme and Non-extreme states

\begin{tabular}{llll} 
Extreme states & Symbol & Non-extreme states & Symbol \\
\hline True & $\mathrm{V}$ & Quasi-true tending to inconsistent & $\mathrm{QV} \rightarrow \mathrm{T}$ \\
False & $\mathrm{F}$ & Quasi-true tending to paracomplete & $\mathrm{QV} \rightarrow \perp$ \\
Inconsistent & $\mathrm{T}$ & Quasi-false tending to inconsistent & $\mathrm{QF} \rightarrow \mathrm{T}$ \\
Paracomplete & $\perp$ & Quasi-false tending to paracomplete & $\mathrm{QF} \rightarrow \perp$ \\
& & Quasi-inconsistent tending to true & $\mathrm{QT} \rightarrow \mathrm{V}$ \\
& & Quasi-inconsistent tending to false & $\mathrm{QT} \rightarrow \mathrm{F}$ \\
& Quasi-paracomplete tending to true & $\mathrm{Q} \perp \rightarrow \mathrm{V}$ \\
& Quasi-paracomplete tending to false & $\mathrm{Q} \perp \rightarrow \mathrm{F}$ \\
\hline
\end{tabular}

Table 2: Paraconsistent artificial neural cells

\begin{tabular}{|c|c|c|c|}
\hline PANC & Inputs & Calculations & Output \\
\hline Analytic connection- $\mathrm{PANC}_{\mathrm{ac}}$ & $\mu, \lambda, \mathrm{Ft}_{\mathrm{ct}}, \mathrm{Ft}_{\mathrm{ce}}$ & $\begin{array}{l}\lambda_{\mathrm{c}}=\mathrm{X}_{\mathrm{c}(\lambda)}(\text { Eq. 3) } \\
\mathrm{G}_{\mathrm{un}}(\text { Eq. } 1) \\
\mathrm{G}_{\mathrm{ce}}(\text { Eq. } 2) \\
\mu_{\mathrm{r}}=\left(\mathrm{G}_{\mathrm{ce}}+1\right) / 2\end{array}$ & $\begin{array}{l}\text { If }\left|\mathrm{G}_{\mathrm{ce}}\right|>\mathrm{Ft}_{\mathrm{ce}} \text { then } \mathrm{S}_{1}=\mu_{\mathrm{r}} \text { and } \mathrm{S}_{2}=0 \\
\text { If }\left|\mathrm{G}_{\mathrm{un}}\right|>\mathrm{Ft}_{\mathrm{ct}} \text { and }\left|\mathrm{G}_{\mathrm{un}}\right|>\left|\mathrm{G}_{\mathrm{ce}}\right| \text { then } \\
\mathrm{S}_{1}=\mu_{\mathrm{r}} \text { and } \mathrm{S}_{2}=\left|\mathrm{G}_{\mathrm{un}}\right| \\
\text { if not } S_{1}=1 / 2 \text { and } S_{2}=0\end{array}$ \\
\hline Maximization-PANC ${ }_{\max }$ & $\mu, \lambda$ & None & If $\mu>\lambda$, then $S_{1}=\mu$, if not $S_{1}=\lambda$ \\
\hline Minimization-PANC $_{\min }$ & $\mu, \lambda$ & None & If $\mu<\lambda$, then $S_{1}=\mu$, if not $S_{1}=\lambda$ \\
\hline
\end{tabular}

Table 3: Group of individuals selected for the study $(\mathrm{p}=0.8496)$

\begin{tabular}{lccr}
\hline & $\begin{array}{l}\text { Normal individuals } \\
\text { control group }\end{array}$ & $\begin{array}{l}\text { Probable AD individuals } \\
\text { AD group }\end{array}$ \\
\hline Male & 8.00 & & 6.00 \\
Female & 26.00 & & 27.00 \\
Mean & 61.38 & 68.00 \\
Schooling & 8.12 & 6.21 \\
MEEM & 24.53 & 20.58 \\
\hline
\end{tabular}

The data acquisition is obtained from magnetic archives (suitable software for physical capture of the signals) or manually (archives TXT-American National Standard Code for Information Interchange). As the actual EEG examination values can vary highly, in module, something like $10 / 1500 \mu \mathrm{V}$, we precede a normalization of the values between 100 and $100 \mu \mathrm{V}$ by a simple linear conversion (Eq. 4), to facilitate the manipulation and to visualize in the screen:

$$
\mathrm{x}=\left(\frac{100 \cdot \mathrm{a}}{\mathrm{m}}\right)
$$

Where:

$\mathrm{m}=$ Maximum value of the exam

$\mathrm{a}=$ Current value of the exam

$\mathrm{x}=$ Current normalized value

It is worth to observe that the process above does not allow the loss of any wave essential characteristics for our analysis.

Elimination of negative cycle: The minimum value of the exam is taken as zero value and the remaining values are translated proportionally.

Data analysis, expert system, and wave morphology: In analyzing EEG signals, one important aspect to take into account is the morphological aspect. To perform this task, it is valuable to build a very simple Expert System, which allows "abnormalities" to be verified, such as spikes and artifacts. Also, it analyses the signal behavior, verifying which band it belongs to (delta, theta, alpha and beta).

Morphological analysis: A control database is composed by waves presenting 256 positions with perfect sinusoidal morphology, with $0.5 \mathrm{~Hz}$ of variance, so taking into account Delta, Theta, Alpha and Beta (of 0.5-30.0 Hz) wave groups.

The process of morphological analysis of a wave is performed by comparing with a certain set of wave patterns (stored in the control database). A wave is associated with a vector (finite sequence of natural numbers) through digital sampling. This vector characterizes a wave pattern and is registered by PANN. Thus, new waves are compared, allowing their recognition or otherwise.

For the sake of completeness, we show some basic aspects of how PANN operates. Let us take three vectors (Fig. 3): V1 = (8, 5, 4, 6, 1); V2 = (8, 6, 4, 6, 5); $\mathrm{V} 3=(8,2,4,6,9)$, where $\mathrm{V} 1$ is the analyzed wave, $\mathrm{V} 2$ and V3 are waves previously stored in the control database. The favorable evidence is calculated as follows: given a pair of vectors, we take ' 1 ' for equal elements and ' 0 ' for different elements, and calculate their percentage.

- Comparing $\mathrm{V}_{2}$ with $\mathrm{V}_{1}: 1+0+1+1+0=3$; in percentage: $(3 / 5) * 100=60 \%$

- Comparing $\mathrm{V}_{3}$ with $\mathrm{V}_{1}: 1+0+1+1+0=3$; in percentage: $(3 / 5) * 100=60 \%$ 
Am. J. Neuroscience 2(1): 17-27, 2011

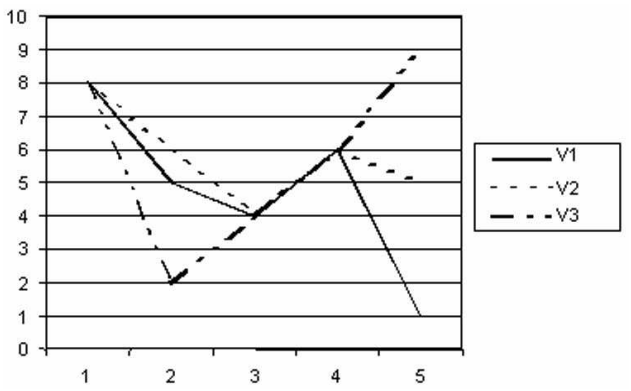

Fig. 3: Comparison of the vectors. Taking as basis the vector $\mathrm{V} 1$, visually we can observe that vector $\mathrm{V} 2$ is 'more similar' to V1 than V3. We use a PANN to recognize this technical system

The contrary evidence is the weighted addition of the differences between the different elements, in module (Eq. 5):

- Comparing $\mathrm{V}_{2}$ with $\mathrm{V}_{1}=0+1 / 10+0+0+4 / 10=$ $(5 / 10) / 5=10 \%$

- Comparing $\mathrm{V}_{3}$ with $\mathrm{V}_{1}=0+3 / 10+0+0+8 / 10=$ $(11 / 10) / 5=22 \%$

$\mathrm{Ce}=\frac{\sum_{\mathrm{j}=1}^{\mathrm{n}}\left(\frac{\left|\mathrm{x}_{\mathrm{j}}-\mathrm{y}_{\mathrm{j}}\right|}{\mathrm{a}}\right)}{\mathrm{n}}$

Where:

$\mathrm{n}=$ Total of elements

$\mathrm{a}=$ Maximum amplitute

$\mathrm{j}=$ Actual element

$\mathrm{Ce}=$ Contrary evidence

Therefore, we can say that $\mathrm{V}_{2}$ is 'more similar' to $\mathrm{V}_{1}$ than $\mathrm{V}_{3}$. We use a PANN to recognize this technical system.

Following this process, PANN was applied successfully in some studies, e.g., speech recognition (Silva Filho et al., 2010).

When the methodology is used in vectors with a huge number of positions, as it is the case of EEG signals, it can present low variance in the favorable evidence.

To avoid this, we introduce other characteristic factor of comparison, the number of peaks of the wave (Eq. 6). In this process, instead we consider as favorable evidence the equality between wave points, we substitute them for the similarity among the peaks of the analyzed waves:

$$
\mathrm{Fe}=1-\left(\frac{(|b d-v t|)}{(b d+v t)}\right)
$$

Where:

$\mathrm{Vt}=$ Number of wave peaks of the exam

$\mathrm{Bd}=$ Number of the wave peaks being compared (pattern stored in the database)

$\mathrm{Fe}=$ Favorable evidence

Each peak is a $1 \mathrm{~Hz}$ morphological approximation; so a wave with 8 peaks is classified as $8 \mathrm{~Hz}$ wave (Alpha band).

At the end of the process, the values of contrary evidence and evidence favorable are submitted to the lattice of decision making. If the coordinated fall on the true region, it is similar to the wave, otherwise as not similar. Therefore, the wave to get more favorable evidence and less contrary evidence will be selected as the most similar wave. Thus, with this improvement we can detect differences among waves more sharply allowing verifying different kinds of interference waveforms (artifacts) and spikes.

In this process, other interesting information can be obtained, the waves' approximate frequency. As the control waves of normality pattern were stored in the database in a systematic way, in other words, with waves with prefixed frequency, then, we know the frequency of each wave. Therefore, when we found the most similar wave to the one that is being analyzed, we also found its frequency. The most amazing advantage of this method of analysis is the low processing, thus it allows using relatively simpler mathematical techniques in comparison with the techniques used nowadays (such as fast Fourier transform).

Data analysis-expert system for detecting the diminishing average frequency level: An expert system verifies the average frequency level of Alpha waves and compares them with a fixed external one (external parameter wave).

Such external parameter can be, for instance, the average frequency of a population or the average frequency of the last exam of the patient. This system also generates two outputs: favorable evidence $\mu$ (normalized values ranging from 0 (corresponds to $100 \%$-or greater frequency loss) to 1 (which corresponds to $0 \%$ of frequency loss) and contrary evidence $\lambda=\mathrm{X}_{\mathrm{c}(\mu)}$ (Eq. 3).

The average frequency of population pattern used in this study is $10 \mathrm{~Hz}$ (Carthery-Goulart et al., 2009).

Data analysis-expert system for high frequency band concentration: This expert system is utilized for Alpha band concentration in the exam. For this, we consider the quotient of the sum of fast Alpha and Beta waves over slow Delta and Theta waves (Eq. 7). This expert system generates two outputs: 
- $\quad$ Favorable evidence $\mu$ (Eq. 7)

- Contrary evidence $\lambda=X_{c(\mu)}$ (Eq. 3)

$\mu=\left(\frac{(\mathrm{A}+\mathrm{B})}{(\mathrm{D}+\mathrm{T})}\right)$

Where:

$\mathrm{A}=$ Alpha band concentration

$\mathrm{B}=$ Beta band concentration

$\mathrm{D}=$ Delta band concentration

$\mathrm{T}=$ Theta band concentration

$\mu=$ Value resulting from the calculation

Data analysis-expert system for low frequency band concentration: This expert system is utilized for Delta band concentration in the exam. For this, we consider the quotient of the sum of slow Delta and Theta waves over fast Alpha and Beta waves (Eq. 8). This expert system generates two outputs:

- $\quad$ Favorable evidence $\mu$ (Eq. 8).

- Contrary evidence $\lambda=X_{c(\mu)}$ (Eq. 3)

$\mu=\left(\frac{(\mathrm{D}+\mathrm{T})}{(\mathrm{A}+\mathrm{B})}\right)$

Where:

$\mathrm{A}=$ Alpha band concentration

$\mathrm{B}=$ Beta band concentration

$\mathrm{D}=$ Delta band concentration

$\mathrm{T}=$ Theta band concentration

$\mu=$ Value resulting from the calculation

Data analysis-decision making: When we analyze information from sources, we may encounter contradictory, fuzzy or para complete data. However, a decision can still be reached. For instance, assuming we have three items of information $\mathrm{PA}, \mathrm{PB}$, and PC, which PA and PB are being analyzed. Thus, if we cannot decide with this expert information, we take the third PC into account in the following way.

The first layer is composed of three analytical PANC connections: $\mathrm{C} 1, \mathrm{C} 2$, and $\mathrm{C} 3$ whose signals are analyzed by means of the Basic Structural EquationBSE (Eq. 9), resulting in the output signals SA, SB and SC (Fig. 4):

$S=\left(\frac{(\mu-(1-\lambda)+1)}{2}\right)$

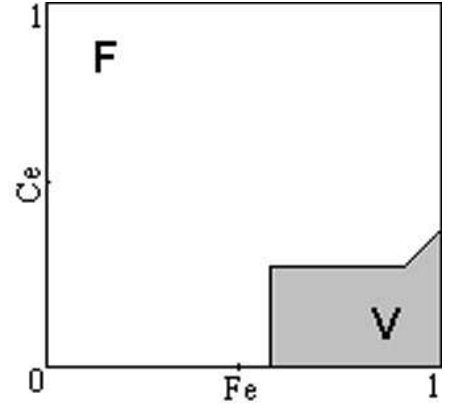

Fig. 4: Lattice of morphological analysis. Ce is the contrary evidence; $\mathrm{Fe}$ is the favorable evidence; $\mathrm{F}$ is logic state False; $\mathrm{V}$ is logic state True

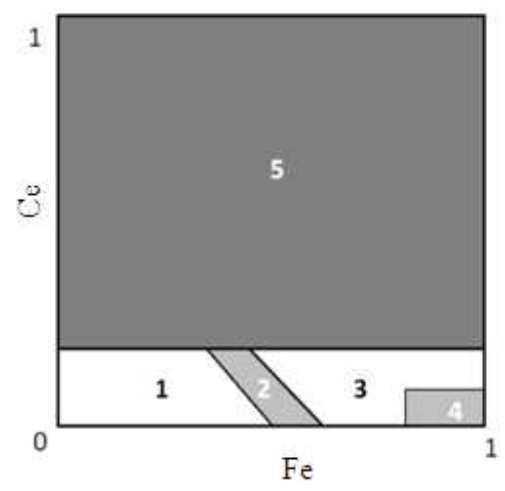

Fig. 5: Lattice of PANN analisys. Ce is the contrary evidence; $\mathrm{Fe}$ is the favorable evidence; $\mathrm{F}$ is logic state False; V is logic state True. Area 1: State logical False (AD likely below average population), 2: State logical Near-real (AD likely than average population); Area 3: StateAlmost logical false (Normal below average population); Area 4: State logical True (Normal above average population); Area 5: logical state of uncertainty (not used in the study area)

In the internal layers, the cells $\mathrm{C} 4$ and $\mathrm{C} 6$ constitute the Maximization Neural Unit (it takes the maximum value SG among output values $\mathrm{SA}, \mathrm{SB}$ and SC) and the cells C5 and C7, the Minimization Neural Unit (which takes the minimum value SE among output values $\mathrm{SA}, \mathrm{SB}$, and $\mathrm{SC}$ ).

To define an interpretation of the analysis is used the resultant value $\left(\mu_{\mathrm{r}}\right)$ and complements, because this generates a complemented resultant value $\left(\lambda_{\mathrm{r}}\right)$. This way, we acquire resultant favorable evidence $\left(\mu_{\mathrm{r}}\right)$ and resultant contrary evidence $\left(\lambda_{\mathrm{r}}\right)$, which are submitted to the lattice of decision making (Fig. 5). 


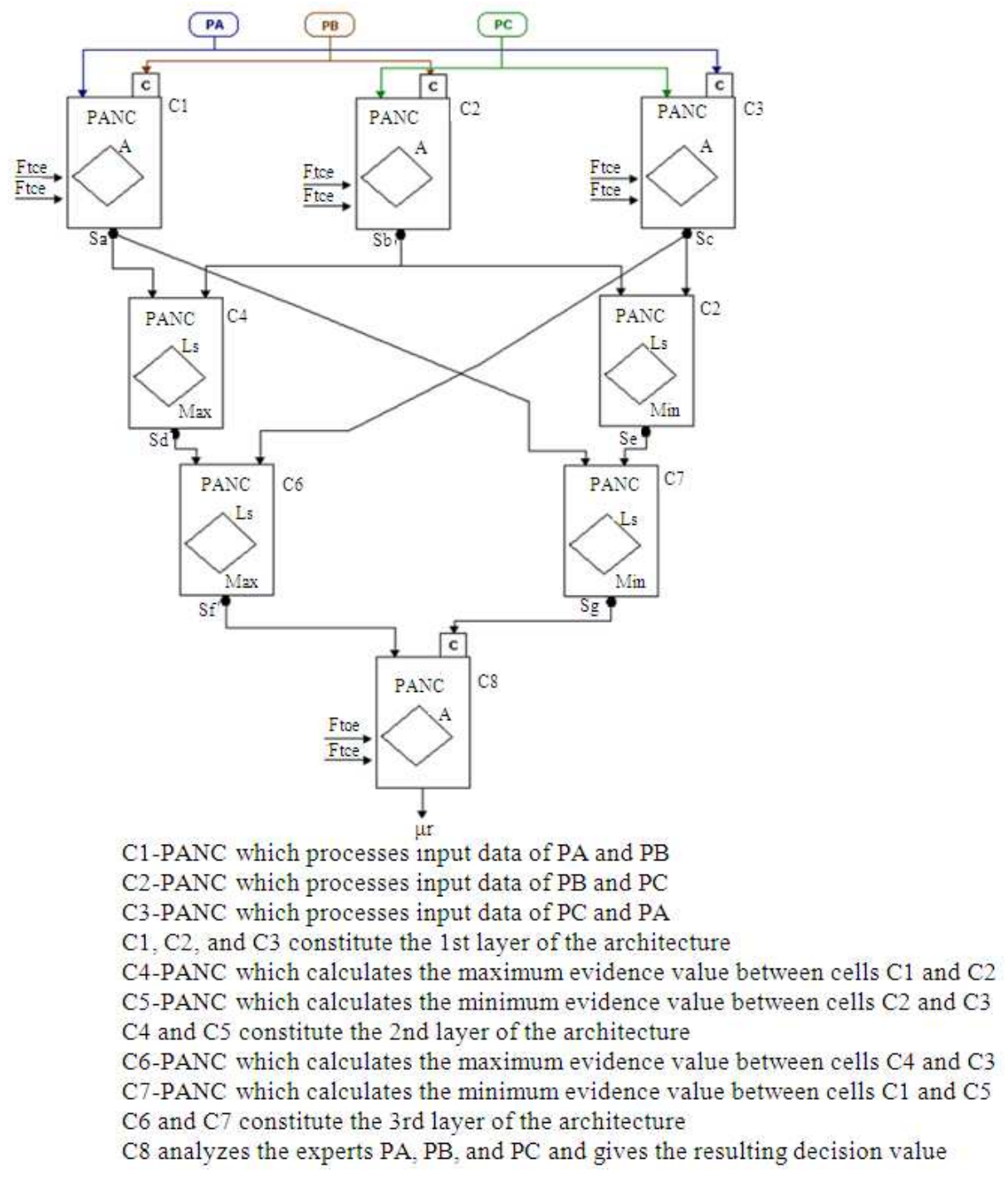

Fig. 6: A decision-making architecture for global analysis. Three expert systems operate: PA, for detecting the diminishing average frequency level; PB, for Alpha band concentration, and PC, for Theta band concentration

\begin{tabular}{|c|c|}
\hline Where: & \\
\hline PANC A & $\begin{array}{l}=\text { Para consistent artificial neural cell of } \\
\text { analytic connection }\end{array}$ \\
\hline PANCLs $_{\text {Max }}$ & $\begin{array}{l}=\text { Para consistent artificial neural cell of } \\
\text { simple logic connection of } \\
\text { maximization }\end{array}$ \\
\hline $\mathrm{PANCLs}_{\mathrm{Min}}$ & $\begin{array}{l}=\text { Para consistent artificial neural cell of } \\
\text { simple logic connection of } \\
\text { minimization }\end{array}$ \\
\hline $\mathrm{Ft}_{\mathrm{ce}}$ & $=$ Certainty tolerance factor \\
\hline $\mathrm{Ft}_{\mathrm{ct}}$ & $=$ Contradiction tolerance factor \\
\hline $\mathrm{S}_{\mathrm{a}}$ & $=$ Output of $\mathrm{C} 1$ cell \\
\hline$S_{b}$ & $=$ Output of $\mathrm{C} 2$ cell \\
\hline$S_{c}$ & $=$ Output of $\mathrm{C} 3$ cell \\
\hline
\end{tabular}

$\begin{array}{ll}\mathrm{S}_{\mathrm{d}} & =\text { Output of C4 cell } \\ \mathrm{S}_{\mathrm{e}} & =\text { Output of C5 cell } \\ \mathrm{S}_{\mathrm{f}} & =\text { Output of C6 cell } \\ \mathrm{S}_{\mathrm{g}} & =\text { Output of C7 cell } \\ \mathrm{C} & =\text { Complemented value of input } \\ \mu_{\mathrm{r}} & =\text { Value of output of PANN }\end{array}$

\section{RESULTS}

The Table 4 and 5 show details of each examination analyzed. The proposed method obtained a sensitivity of $80 \%$ and a specificity of $73 \%$, as shown in Table 6. Figure 8 shows the distribution of lattice results in decision-making. 
Am. J. Neuroscience 2(1): 17-27, 2011

Table 4: Test with normal patients. $\mathrm{FE}=$ Favorable Evidence; $\mathrm{CE}=$ Contrary Evidence; Diagnosis $-1=$ Normal individual $2=$ Probable AD patient; Delta, Theta, Alpha, and Beta = quantity of waves; Mean = Mean of quantity of waves

\begin{tabular}{|c|c|c|c|c|c|c|c|c|c|}
\hline Exam & Patient & Delta & Theta & Alpha & Beta & Mean & FE & $\mathrm{CE}$ & Diagnosis \\
\hline 7601 & JS & 7 & 152 & 111 & 0 & 6.91840 & 0.4813 & 0.1404 & 1 \\
\hline 7701 & RKG & 0 & 100 & 215 & 24 & 8.47500 & 0.4813 & 0.0712 & 2 \\
\hline 5401 & $\mathrm{EC}$ & 4 & 157 & 108 & 12 & 7.02500 & 0.4959 & 0.1377 & 2 \\
\hline 7801 & JIS & 1 & 91 & 209 & 39 & 8.50000 & 0.5191 & 0.0603 & 1 \\
\hline 6501 & LANG & 4 & 86 & 222 & 25 & 8.42500 & 0.5207 & 0.0548 & 1 \\
\hline 7101 & JTBT & 0 & 89 & 243 & 12 & 8.60000 & 0.5419 & 0.0594 & 1 \\
\hline 7201 & OTWNV & 0 & 74 & 249 & 13 & 8.40000 & 0.5896 & 0.0301 & 1 \\
\hline 1202 & RA & 6 & 44 & 194 & 164 & 10.20000 & 0.8162 & 0.0613 & 1 \\
\hline 2102 & DYT & 7 & 66 & 101 & 579 & 18.82500 & 0.8546 & 0.0485 & 1 \\
\hline 1802 & DO & 0 & 32 & 269 & 105 & 10.15000 & 0.8818 & 0.0394 & 1 \\
\hline 6101 & EFRC & 0 & 31 & 261 & 106 & 10.19860 & 0.8832 & 0.0389 & 2 \\
\hline 1902 & ILM & 3 & 26 & 242 & 136 & 10.48420 & 0.8931 & 0.0356 & 1 \\
\hline 3001 & $\mathrm{AB}$ & 10 & 27 & 40 & 584 & 16.52500 & 0.9580 & 0.0280 & 2 \\
\hline 1605 & DO & 0 & 21 & 308 & 88 & 10.42500 & 0.9622 & 0.0252 & 1 \\
\hline 1303 & DO & 2 & 12 & 308 & 74 & 10.16440 & 0.9735 & 0.0177 & 1 \\
\hline 2202 & GM & 0 & 39 & 93 & 1064 & 29.90000 & 0.9755 & 0.0163 & 2 \\
\hline 2001 & LBA & 2 & 19 & 82 & 508 & 17.07500 & 0.9769 & 0.0154 & 1 \\
\hline 5901 & DG & 0 & 13 & 181 & 258 & 11.88330 & 0.9784 & 0.0144 & 1 \\
\hline 1103 & DO & 0 & 12 & 259 & 150 & 10.81310 & 0.9786 & 0.0143 & 1 \\
\hline 2401 & NAG & 2 & 7 & 285 & 108 & 10.07500 & 0.9833 & 0.0112 & 1 \\
\hline 1004 & $\mathrm{ON}$ & 0 & 14 & 102 & 562 & 16.95000 & 0.9845 & 0.0103 & 1 \\
\hline 2302 & GAA & 0 & 11 & 168 & 429 & 16.31000 & 0.9864 & 0.0090 & 1 \\
\hline 1404 & RA & 0 & 7 & 316 & 78 & 10.02500 & 0.9869 & 0.0087 & 1 \\
\hline 2901 & LFM & 2 & 15 & 87 & 923 & 26.70260 & 0.9876 & 0.0083 & 1 \\
\hline 2701 & AEJO & 2 & 12 & 99 & 995 & 29.94440 & 0.9905 & 0.0063 & 1 \\
\hline 1604 & MLSD & 3 & 7 & 141 & 720 & 25.98125 & 0.9914 & 0.0057 & 2 \\
\hline 2201 & MHA & 0 & 0 & 101 & 941 & 26.05000 & 1.0000 & 0.0000 & 1 \\
\hline 2501 & YVG & 0 & 0 & 0 & 1347 & 34.30260 & 1.0000 & 0.0000 & 1 \\
\hline 4001 & TANB & 15 & 135 & 98 & 26 & 6.85000 & 0.5107 & 0.1162 & 2 \\
\hline 1201 & $\mathrm{E}$ & 4 & 32 & 175 & 238 & 11.50000 & 0.8797 & 0.0401 & 1 \\
\hline 1704 & JSM & 0 & 25 & 231 & 195 & 11.27500 & 0.9584 & 0.0277 & 1 \\
\hline 2103 & MRA & 0 & 30 & 108 & 407 & 14.34210 & 0.9587 & 0.0275 & 1 \\
\hline 1503 & $\mathrm{ACP}$ & 4 & 5 & 327 & 39 & 9.37500 & 0.9664 & 0.0193 & 2 \\
\hline 1302 & MM & 4 & 0 & 161 & 474 & 15.97500 & 0.9953 & 0.0031 & 1 \\
\hline 4301 & NGP & 15 & 153 & 103 & 0 & 6.77500 & 0.4544 & 0.1487 & 1 \\
\hline 7501 & IOG & 13 & 161 & 71 & 37 & 7.05000 & 0.4635 & 0.1610 & 1 \\
\hline 3201 & GBS & 4 & 40 & 264 & 50 & 8.95000 & 0.7631 & 0.0090 & 2 \\
\hline 1203 & CLD & 3 & 42 & 286 & 26 & 9.16050 & 0.7690 & 0.0211 & 2 \\
\hline 2601 & RPS & 6 & 47 & 141 & 291 & 12.56710 & 0.8361 & 0.0546 & 2 \\
\hline 3101 & JCS & 0 & 46 & 223 & 134 & 10.07500 & 0.8288 & 0.0571 & 2 \\
\hline 2101 & MW & 7 & 75 & 196 & 68 & 8.65000 & 0.5770 & 0.0510 & 2 \\
\hline
\end{tabular}

\section{DISCUSSION}

We believe that a process of the examination analysis using a PANN attached to EEG findings, such as relations between frequency bandwidth and inter hemispheric coherences, can create computational methodologies that allow the automation of analysis and diagnosis. The computational implementation of PANN shown in Fig. 6 can be performed very easily, thus enabling their application.

As seen in Fig. 7, the method can distinguish groups and subgroups of individuals. Both in relation to normal or probable $\mathrm{AD}$, as for the average number of individuals, ie, the method can differentiate normal patients from probable $\mathrm{AD}$ patients regardless of the average frequency of brain activity of the individual.
These methodologies could be employed as tools to aid in the diagnosis of diseases such as Alzheimer's disease, provided they have defined electroencephalographic findings.

In the case of Alzheimer's disease, for example, in studies carried out previously (Lopes et al., 2009) shown satisfactory results (but still far from being a tool to aid clinical) that demonstrated the computational efficiency of the methodology using a simple morphological analysis (only Paraconsistent Annotated Logic $\mathrm{E} \tau$ ). These results encouraged us to improve the morphological analysis of the waves and try to apply the method in other diseases besides Alzheimer's disease.

With the process of morphological analysis using the PANN, it becomes possible to quantify the frequency average of the individual without losing its temporal reference. 
Am. J. Neuroscience 2(1): 17-27, 2011

Table 5: Test with non-normal patients FE = Favorable Evidence; $\mathrm{CE}=$ Contrary Evidence; Diagnosis $-1=$ Normal individual, $2=$ Probable AD patient; Delta, Theta, Alpha, and Beta = quantity of waves; Mean = Mean of quantity of waves

\begin{tabular}{|c|c|c|c|c|c|c|c|c|c|}
\hline Exam & Patient & Delta & Theta & Alpha & Beta & Mean & $\mathrm{FE}$ & $\mathrm{CE}$ & Diagnosis \\
\hline 4101 & MTRS & 6 & 104 & 168 & 24 & 7.5500 & 0.3311 & 0.0596 & 2 \\
\hline 6001 & EGT & 8 & 177 & 40 & 0 & 5.9210 & 0.4373 & 0.2072 & 2 \\
\hline 7901 & AMNT & 5 & 71 & 162 & 147 & 9.6250 & 0.6851 & 0.0800 & 1 \\
\hline 5701 & $\mathrm{ABC}$ & 6 & 55 & 202 & 120 & 9.5750 & 0.7398 & 0.0584 & 2 \\
\hline 2203 & JPNF & 11 & 142 & 94 & 0 & 6.1750 & 0.1204 & 0.1185 & 2 \\
\hline 6201 & ESSE & 0 & 144 & 146 & 12 & 7.5500 & 0.1623 & 0.1159 & 2 \\
\hline 6301 & $\mathrm{MF}$ & 0 & 137 & 162 & 0 & 7.4750 & 0.1865 & 0.1028 & 2 \\
\hline 7301 & AOFFS & 10 & 117 & 144 & 27 & 7.4500 & 0.2332 & 0.0856 & 1 \\
\hline 5501 & TMOG & 16 & 155 & 62 & 13 & 6.1500 & 0.2352 & 0.1551 & 2 \\
\hline 6401 & RRS & 4 & 176 & 72 & 0 & 6.3000 & 0.2564 & 0.1721 & 2 \\
\hline 8102 & ABS & 0 & 123 & 168 & 27 & 7.9500 & 0.3173 & 0.0909 & 2 \\
\hline 5801 & TCS & 15 & 177 & 47 & 13 & 6.3000 & 0.3279 & 0.1960 & 1 \\
\hline 1504 & CLD & 11 & 96 & 203 & 0 & 7.7500 & 0.3698 & 0.0601 & 1 \\
\hline 8001 & BLW & 4 & 114 & 174 & 40 & 8.3000 & 0.3819 & 0.0927 & 1 \\
\hline 1703 & CLD & 4 & 104 & 208 & 0 & 7.9000 & 0.3823 & 0.0659 & 2 \\
\hline 1801 & ZSA & 4 & 101 & 187 & 16 & 7.8907 & 0.3832 & 0.0650 & 2 \\
\hline 2801 & CRSV & 8 & 89 & 213 & 13 & 8.0750 & 0.4533 & 0.0539 & 2 \\
\hline 43901 & AVB & 8 & 152 & 114 & 12 & 7.1500 & 0.5092 & 0.1372 & 2 \\
\hline 44001 & ASS & 40 & 165 & 8 & 0 & 5.4552 & 0.6709 & 0.2540 & 2 \\
\hline 1701 & LHO & 4 & 64 & 242 & 59 & 9.2250 & 0.6848 & 0.0534 & 2 \\
\hline 1102 & MLCM & 6 & 67 & 202 & 107 & 9.5500 & 0.6909 & 0.0730 & 2 \\
\hline 1702 & RF & 0 & 65 & 227 & 81 & 9.3250 & 0.7049 & 0.0534 & 2 \\
\hline 1301 & MGC & 7 & 66 & 148 & 216 & 11.5000 & 0.7494 & 0.0835 & 2 \\
\hline 1606 & OSP & 0 & 63 & 214 & 121 & 10.1723 & 0.7626 & 0.0791 & 2 \\
\hline 4201 & MAP & 8 & 43 & 221 & 115 & 9.6750 & 0.7861 & 0.0496 & 1 \\
\hline 1803 & $\mathrm{ABM}$ & 4 & 54 & 191 & 171 & 10.5000 & 0.7929 & 0.0690 & 2 \\
\hline
\end{tabular}

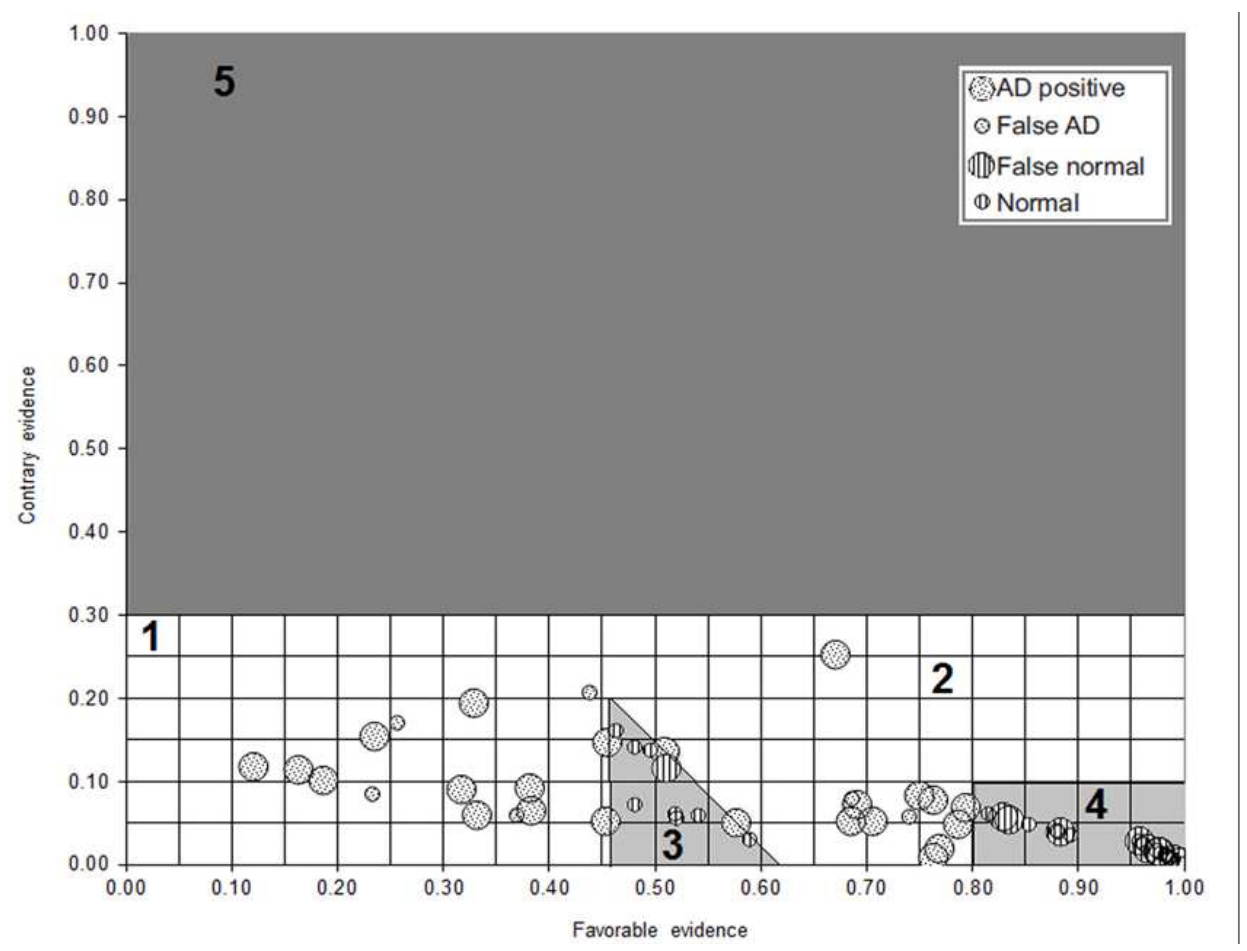

Fig. 7: The lattice final decision of the review process of PANN with the result of the 67 examinations. Area 1: State logical False (AD likely below average population), 2: State logical Near-real (AD likely than average population); Area 3: State-Almost logical false (Normal below average population); Area 4: State logical True (Normal above average population); Area 5: logical state of uncertainty (not used in the study area) 
Am. J. Neuroscience 2(1): 17-27, 2011

Table 6: Diagnosis-normal individual x probable AD patients

Gold standard

\begin{tabular}{llll} 
& & AD Patient (\%) & Notal (\%) \\
\hline \multirow{2}{*}{ PANN } & AD Patient & 35.82 & 14.93 \\
& Normal individual & 8.96 & 40.30 \\
& Total & 44.78 & 55.22 \\
& Sensitivity: & 0.80 & 100.00 \\
Specificity: & 0.73 & \\
Index of coincidence (Kappa): & 0.76 & \\
\hline
\end{tabular}

This feature becomes a differential, compared to traditional analysis of quantification of frequencies, such as Fast Fourier Transform (FFT), aiming at a future application in real-time analysis, i.e. at the time of acquisition of the EEG exams.

For this future application, it must be assumed that the automatic detection of spikes and artifacts are important functions that should be aggregated for analysis, thus creating variations in morphology specialized detection devices, for example.

It is noteworthy that by treating the PANN a relatively new theory and extend the operation of classical PANN is justified to use different approaches (as discussed in this study) to know the full potential of the theory applied to the specific and real needs.

\section{CONCLUSION}

The methodology of pattern of recognition (through PANNs) using morphological analysis showed itself to be effective, achieving recognize patterns of waves similar to patterns stored in the database. In addition, this methodology allows the quantification and qualification of the examination of EEG data to be used by PANN in its process of examination analysis. PANN also proved to be an agile and promising as a tool for distinguishing among patients, providing a satisfactory performance, classifying them with good sensitivity but low specificity.

The setup possibilities allows PANN to make further studies with larger number of patients, and then our findings could be used as basic values to achieve new comparisons. The characteristics of Para consistent Logic and PANNs show up effective in recognizing patterns. Moreover, our results may extend to other studies of waves, such as identification of artifacts and also to other diseases in which EEG can be used as a clinical procedure. Finally, our study opens opportunities for future studies using other options for processing and treating the EEG signals with Paraconsistent Logic and PANN.

\section{ACKNOWLEDGEMENT}

The researchers are grateful to the anonymous referees providing useful comments to improving this version of the study.

\section{REFERENCES}

Abe, J.M. and K. Nakamatsu, 2009. A survey of paraconsistent annotated logics and applications. Int. J. Reason. Intell. Syst., 1: 31-42. DOI: 10.1504/IJRIS.2009.026715

Abe, J.M., H.F.S. Lopes and R. Anghinah, 2011. Paraconsistent artificial neural networks and EEG analysis. Int. J. Reason. Intell. Syst., 3: 115-123. DOI: 10.1504/IJRIS.2011.042266

Carthery-Goulart, M.T., R. Anghinah, R. ArezaFegyveres, V.S. Bahia and S.M. Brucki et al., 2009. Performance of a Brazilian population on the test of functional health literacy in adults. Rev. Saude Publica., 43: 631-638. PMID: 19488667

Duffy, F.H., G.B. McAnulty, M.C. McCreary, G.J. Cuchural and A.L. Komaroff, 2011. EEG spectral coherence data distinguish chronic fatigue syndrome patients from healthy controls and depressed patients-A case control study. BMC Neurol., 11: 82-82. DOI: 10.1186/1471-2377-1182 PMID: 21722376

Karait, N.O.S.B., S.M. Shamsuddin and R. Sudirman, 2009. Swarm negative selection algorithm for electroencephalogram signals classification. J. Comput. Sci., 5: 995-1002. DOI: 10.3844/jcssp.2009.995.1002

Lopes, H.F.S., J.M. Abe and R. Anghinah, 2009. Application of paraconsistent artificial neural networks as a method of aid in the diagnosis of alzheimer disease. J. Med. Syst., 34: 1073-1081. DOI: 10.1007/s10916-009-9325-2

Machado, S., F. Araujo, F. Paes, B. Velasques and M. Cunha et al., 2010. EEG-based brain-computer interfaces: An overview of basic concepts and clinical applications in neurorehabilitation. Rev. Neurosci., 21: 451-468. PMID: 21438193 
Puri, I.K. and L. Li, 2010. Mathematical Modeling for the Pathogenesis of Alzheimer's disease. PLoS ONE, 5: e15176-e15176. DOI: 10.1371/journal.pone.0015176 PMID: 21179474

Silva Filho, J.I.D., G.L. Torres and J.M. Abe, 2010. Uncertainty Treatment Using Paraconsistent Logic: Introducing Paraconsistent Artificial Neural Networks. 1st Edn., Ios Pr Inc, IOS Press, Amsterdam, Washington, DC., ISBN: 9781607505570, pp: 311.
Syan, C.S. and R.E.S. Harnarinesingh, 2010. Comparison of pre-processing and classification techniques for single-trial and multi-trial p300based brain computer interfaces. Am. J. Applied Sci., $\quad 7$ : 1219-1225. DOI: 10.3844/ajassp.2010.1219.1225 